\title{
Existence of positive periodic solutions of second-order differential equations with weak singularities
}

You Ma*

\section{"Correspondence:}

mayou1225@163.com

School of Mathematical Sciences,

Beijing Normal University, Beijing

100875, P.R. China

\begin{abstract}
We establish the existence of positive periodic solutions of the second-order differential equation $x^{\prime \prime}+a(t) x=f(t, x)+c(t)$ via Schauder's fixed point theorem, where $a \in L^{1}\left(\mathbb{R} / T \mathbb{Z} ; \mathbb{R}_{+}\right), c \in L^{1}(\mathbb{R} / T \mathbb{Z} ; \mathbb{R}), f$ is a Caratheodory function and it is singular at $x=0$. Our main results generalize some recent results by Torres (J. Differ. Equ. 232:277-284, 2007).

MSC: 34B10; 34B18

Keywords: periodic solution; weak singularity; Schauder's fixed point theorem; indefinite weight
\end{abstract}

\section{Introduction}

In this paper, we are concerned with the existence of positive periodic solutions of the second-order differential equation

$$
x^{\prime \prime}+a(t) x=f(t, x)+c(t)
$$

with $a \in L^{1}\left(\mathbb{R} / T \mathbb{Z} ; \mathbb{R}_{+}\right), c \in L^{1}(\mathbb{R} / T \mathbb{Z} ; \mathbb{R}), f \in \operatorname{Car}(\mathbb{R} / T \mathbb{Z} \times(0, \infty) ; \mathbb{R})$ is a $L^{1}$ Caratheodory function, and $f$ is singular at $x=0$.

The interest on this type of equations began with the paper of Lazer and Solimini [1]. They dealt with the case that $a \equiv 0$ and $f(x)=\frac{1}{x^{\lambda}},(1.1)$ reduces to the special equation

$$
x^{\prime \prime}=\frac{1}{x^{\lambda}}+c(t)
$$

which was initially studied by Lazer and Solimini [1]. They proved that for $\lambda \geq 1$ (called a strong force condition in the terminology first introduced by Gordon [2, 3]), a necessary and sufficient condition for the existence of a positive periodic solution of (1.2) is that the mean value of $c$ is negative,

$$
\bar{c}=\frac{1}{T} \int_{0}^{T} c(t) d t<0
$$

Moreover, if $0<\lambda<1$ (weak force condition) they found examples of functions $c$ with negative mean values and such that periodic solutions do not exist.

(c) 2014 Ma; licensee Springer. This is an Open Access article distributed under the terms of the Creative Commons Attribution License (http://creativecommons.org/licenses/by/4.0), which permits unrestricted use, distribution, and reproduction in any medium, provided the original work is properly credited. 
If compared with the literature available for strong singularities, see [4-15] and the references therein, the study of the existence of periodic solutions in the presence of a weak singularity is much more recent and the number of references is considerably smaller. The likely reason may be that with a weak singularity, the energy near the origin becomes finite, and this fact is not helpful for obtaining the a priori bound needed for a classical application of the degree theory, and also is not helpful for the fast rotation needed in recent versions of the Poincaré-Birkhoff theorem. The first existence result with a weak force condition appears in Rachunková et al. [16]. Since then, Eq. (1.1) with $f$ having weak singularities has been studied by several authors; see Torres $[17,18]$, Franco and Webb [19], Chu and Li [20] and Li and Zhang [21].

Recently, Torres [18] showed how a weak singularity can play an important role if Schauder's fixed point theorem is chosen in the proof of the existence of positive periodic solutions for (1.1). From now on, for a given function $\xi \in L^{\infty}[0, \infty]$, we denote the essential supremum and infimum of $\xi$ by $\xi^{*}$ and $\xi_{*}$, respectively. We write $\xi \succ 0$ if $\xi \geq 0$ for a.e. $t \in[0, T]$ and it is positive in a set of positive measure. Under the following assumption:

(H1) the linear equation $u^{\prime \prime}+a(t) u=0$ is nonresonant and the corresponding Green's function obeys

$$
G(t, s) \geq 0, \quad(t, s) \in[0, T] \times[0, T]
$$

Torres showed the following.

Theorem A [18, Theorem 1] Let (H1) hold and define

$$
\gamma(t)=\int_{0}^{T} G(t, s) c(s) d s
$$

\section{Assume that}

(H2) there exist $b \in L^{1}(0, T)$ with $b \succ 0$ and $\lambda>0$ such that

$$
0 \leq f(t, u) \leq \frac{b(t)}{u^{\lambda}} \text { for all } u>0 \text {, a.e. } t \in[0, T]
$$

If $\gamma_{*}>0$, then there exists a positive T-periodic solution of (1.1).

\section{Theorem B [18, Theorem 2] Let (H1) hold. Assume that}

(H3) there exist two functions $b, \hat{b} \in L^{1}(0, T)$ with $b, \hat{b} \succ 0$ and a constant $\lambda \in(0,1)$ such that

$$
0 \leq \frac{\hat{b}(t)}{u^{\lambda}} \leq f(t, u) \leq \frac{b(t)}{u^{\lambda}}, \quad u \in(0, \infty) \text {, a.e. } t \in[0, T]
$$

If $\gamma_{*}=0$, then (1.1) has a positive T-periodic solution.

Theorem C [18, Theorem 4] Let (H1) and (H3) hold. Let

$$
\hat{\beta}_{*}=\min _{t \in[0, T]}\left(\int_{0}^{T} G(t, s) \hat{b}(s) d s\right), \quad \beta^{*}=\max _{t \in[0, T]}\left(\int_{0}^{T} G(t, s) b(s) d s\right) .
$$




$$
\begin{aligned}
& \text { If } \gamma^{*} \leq 0 \text { and } \\
& \qquad \gamma_{*} \geq\left(\frac{\hat{\beta}_{*}}{\left(\beta^{*}\right)^{\lambda}} \lambda^{2}\right)^{\frac{1}{1-\lambda^{2}}}\left(1-\frac{1}{\lambda^{2}}\right),
\end{aligned}
$$

then (1.1) has a positive T-periodic solution.

Obviously, (H2) and (H3) are too restrictive so that the above mentioned results are only applicable to (1.1) with nonlinearity which is bounded at origin and infinity by a function of the form $\frac{1}{u^{\lambda}}$. Very recently, Ma et al. [22] generalized Theorems A-C under some conditions which allow the nonlinearity $f$ to be bounded by two different functions $\frac{1}{u^{\alpha}}$ and $\frac{1}{u^{\beta}}$. Notice that $b \succ 0$ in (H2), and $b, \hat{b} \succ 0$ in (H3). Of course the natural question is: what would happen if we allow that the functions $b$ and $\hat{b}$ may change sign?

It is worth remarking that if $b \in L^{1}(\mathbb{R} / T \mathbb{Z} ; \mathbb{R})$ changes its sign, then the existence of $T$-periodic solutions for the equation

$$
x^{\prime \prime}=\frac{b(t)}{x^{3}}
$$

is still open; see Bravo and Torres [23] and Hakl and Torres [24]. Notice that (1.4) plays an important role in the study of stabilization of matter-wave breathers in Bose-Einstein condensates [25], the propagation of guided waves in optical fibers [26], and in the electromagnetic trapping of a neutral atom near a charged wire [27].

In the sequel, we denote the set of continuous $T$-periodic functions by $C_{T}$. Define

$$
\gamma(t)=\int_{0}^{T} G(t, s) c(s) d s
$$

which is just the unique $T$-periodic solution of the linear equation $x^{\prime \prime}+a(t) x=c(t)$. For $y \in C[0, T]$, let us define

$$
y^{+}=\max \{y, 0\}, \quad y^{-}=\max \{-y, 0\} .
$$

It is the purpose of this paper to general Theorems A-C under some assumptions which allow the nonlinearity $b$ and $\hat{b}$ to change sign. The main tool is Schauder's fixed point theorem.

\section{Existence of periodic solutions}

We will state and prove three results on the existence of $T$-periodic positive solutions of (1.1) according to the cases

$$
\gamma^{*}>0 ; \quad \gamma^{*}=0 ; \quad \gamma^{*}<0
$$

Theorem 2.1 Let $\gamma^{*}>0$. Assume $(\mathrm{H} 1)$ and

(A1) there exists $b \in C[0, T]$ with

$$
\text { meas } I_{+}>0, \quad \text { meas } I_{-}>0 \text {, }
$$


where

$$
I_{+}=\{t \in[0, T] \mid b(t)>0\}, \quad I_{-}=\{t \in[0, T] \mid b(t)<0\},
$$

and there exists $\lambda>0$ such that

$$
0 \leq f(t, x) \leq \frac{b(t)}{x^{\lambda}}, \quad \forall x \in(0, \infty) \text { and } t \in I_{+}
$$

and

$$
0 \geq f(t, x) \geq \frac{b(t)}{x^{\lambda}}, \quad \forall x \in(0, \infty) \text { and } t \in I_{-} .
$$

If

$$
\left(\beta^{-}\right)^{*} \leq\left(\frac{\gamma_{*}}{2}\right)^{1+\lambda}
$$

then there exists a positive T-periodic solution of (1.1).

Proof A T-periodic solution of (1.1) is just a fixed point of the completely continuous map $\mathcal{F}: C_{T} \rightarrow C_{T}$ defined as

$$
\mathcal{F}[u](t):=\int_{0}^{T} G(t, s)[f(s, x(s))+c(s)] d s=\int_{0}^{T} G(t, s) f(s, x(s)) d s+\gamma(t) .
$$

By a direct application of Schauder's fixed point theorem, the proof is finished if we prove that $\mathcal{F}$ maps the closed convex set defined as

$$
K=\left\{x \in C_{T}: r \leq x(t) \leq R \text { for all } t\right\}
$$

into itself, where $R>r>0$ are positive constants to be fixed properly. In fact, if we define the function

$$
\beta^{+}(t)=\int_{0}^{T} G(t, s) b^{+}(s) d s, \quad \beta^{-}(t)=\int_{0}^{T} G(t, s) b^{-}(s) d s,
$$

let us fix $r:=\frac{\gamma_{*}}{2}$ which is positive by hypothesis. Given $x \in K$, by the nonnegative sign of $G$ and (2.4)-(2.5),

$$
\begin{aligned}
& \mathcal{F}[u](t) \geq \frac{\left(\beta^{+}\right)_{*}}{R^{\lambda}}-\frac{\left(\beta^{-}\right)^{*}}{r^{\lambda}}+\gamma_{*} \geq-\frac{\left(\beta^{-}\right)^{*}}{r^{\lambda}}+\gamma_{*}, \\
& \mathcal{F}[u](t) \leq \frac{\left(\beta^{+}\right)^{*}}{r^{\lambda}}-\frac{\left(\beta^{-}\right)_{*}}{R^{\lambda}}+\gamma^{*} \leq \frac{\left(\beta^{+}\right)^{*}}{r^{\lambda}}+\gamma^{*} .
\end{aligned}
$$

Set

$$
r:=\frac{\gamma_{*}}{2}, \quad R:=\frac{\left(\beta^{+}\right)^{*}}{\left(\gamma_{*} / 2\right)^{\lambda}}=\frac{2^{\lambda}\left(\beta^{+}\right)^{*}}{\gamma_{*}^{\lambda}}+\gamma^{*}
$$


Then for $x \in K$, it follows from (2.6) that

$$
\mathcal{F}[u](t) \geq \frac{\gamma_{*}}{2}=r
$$

and

$$
\mathcal{F}[u](t) \leq \frac{2^{\lambda}\left(\beta^{+}\right)^{*}}{\gamma_{*}^{\lambda}}+\gamma^{*}=R
$$

Thus, $\mathcal{F}(K) \subset K$. Clearly, $R>r>0$, so the proof is finished.

Remark 2.1 If $b \succ 0$ in $[0, T]$, then $\beta^{-} \equiv 0$, and accordingly $\left(\beta^{-}\right)_{*}=0$. In this case, (2.6) is satisfied for all $\lambda>0$ and $\gamma$ with $\gamma_{*}>0$. So, Theorem 2.1 generalizes Theorem A.

For $u \in C_{T}$, let us define

$$
I_{+}^{u}=\{t \in[0, T] \mid u(t)>0\}, \quad I_{-}^{u}=\{t \in[0, T] \mid u(t)<0\} .
$$

Theorem 2.2 Let $\gamma_{*}=0$. Assume $(\mathrm{H} 1)$ and

(A2) there exist $b, h \in C[0, T]$ and $0<\lambda<1$ such that

$$
\begin{aligned}
& I_{+}^{h} \subseteq I_{+}^{b}, \quad I_{-}^{h} \subseteq I_{-}^{b}, \\
& 0 \leq \frac{h(t)}{x^{\lambda}} \leq f(t, x) \leq \frac{b(t)}{x^{\lambda}}, \quad \forall x \in(0, \infty) \text { and a.e. } t \in I_{+}^{b}, \\
& 0 \geq \frac{h(t)}{x^{\lambda}} \geq f(t, x) \geq \frac{b(t)}{x^{\lambda}}, \quad \forall x \in(0, \infty) \text { and a.e. } t \in I_{-}^{b},
\end{aligned}
$$

(A3) $h$ and b satisfy

$$
\left(h^{+}\right)_{*} s_{0}^{1-\lambda}-\left(b^{-}\right)^{*} s_{0}^{\lambda+1} \geq 1,
$$

where

$$
s_{0}:=\min \left\{s \mid s \in[2, \infty) \text { with }\left(h^{+}\right)_{*} s^{1-\lambda} \geq 1 \text { and }\left(b^{+}\right)^{*} s^{\lambda}+\gamma^{*} \leq s\right\} .
$$

Then there exists a positive T-periodic solution of (1.1).

Proof We follow the same strategy and notation as in the proof of Theorem 2.1. Again, we need to fix $r<R$ such that $F(K) \subseteq K$. We define the functions

$$
\begin{array}{ll}
\beta^{+}(t)=\int_{0}^{T} G(t, s) b^{+}(s) d s, & \beta^{-}(t)=\int_{0}^{T} G(t, s) b^{-}(s) d s, \\
\delta^{+}(t)=\int_{0}^{T} G(t, s) h^{+}(s) d s, & \delta^{-}(t)=\int_{0}^{T} G(t, s) h^{-}(s) d s .
\end{array}
$$

Recall

$$
\mathcal{F}[u](t):=\int_{0}^{T} G(t, s)[f(s, x(s))+c(s)] d s=\int_{0}^{T} G(t, s) f(s, x(s)) d s+\gamma(t) .
$$


Some easy computations prove that it is sufficient to find $r<R$ such that

$$
\begin{aligned}
\mathcal{F}[u](t) & \leq \int_{I_{+}^{b}} G(t, s) \frac{b^{+}(s)}{r^{\lambda}} d s-\int_{I_{-}^{h}} G(t, s) \frac{h^{-}(s)}{R^{\lambda}} d s+\gamma^{*} \\
& \leq \frac{\left(b^{+}\right)^{*}}{r^{\lambda}}-\frac{\left(h^{-}\right)_{*}}{R^{\lambda}}+\gamma^{*} \leq R, \\
\mathcal{F}[u](t) & \geq \int_{I_{+}^{h}} G(t, s) \frac{h^{+}(s)}{R^{\lambda}} d s-\int_{I_{-}^{b}} G(t, s) \frac{b^{-}(s)}{r^{\lambda}} d s \\
& \geq \frac{\left(h^{+}\right)_{*}}{R^{\lambda}}-\frac{\left(b^{-}\right)^{*}}{r^{\lambda}} \geq r .
\end{aligned}
$$

Taking $R=1 / r$, it is sufficient to find $R>1$ such that

$$
\begin{aligned}
& \left(b^{+}\right)^{*} R^{\lambda}-\frac{\left(h^{-}\right)_{*}}{R^{\lambda}}+\gamma^{*} \leq R, \\
& \left(h^{+}\right)_{*} R^{1-\lambda}-\left(b^{-}\right)^{*} R^{\lambda+1} \geq 1 .
\end{aligned}
$$

Obviously, conditions (2.15) and (2.16) guarantee that (2.20) and (2.21) hold for $R=s_{0}$.

Theorem 2.3 Let $\gamma^{*} \leq 0$. Assume (H1) and (A2) and

(A4) there exists

$$
r_{0} \in\left\{r \mid r \in(0,1 / 2] \text { and } r^{\lambda} \leq\left(b^{+}\right)^{*} \text { and } \frac{\left(h^{+}\right)_{*}}{\left[\left(b^{+}\right)^{*}\right]^{\lambda}} r^{\lambda^{2}}+\gamma_{*} \geq r\right\}
$$

such that

$$
\frac{\left(h^{+}\right)_{*}}{\left[\left(b^{+}\right)^{*}\right]^{\lambda}} r_{0}^{\lambda^{2}}-\frac{\left(b^{-}\right)^{*}}{r_{0}^{\lambda}}+\gamma_{*} \geq r_{0} .
$$

Then there exists a positive T-periodic solution of (1.1).

Proof In this case, to prove that $\mathcal{F}(K) \subset K$ it is sufficient to find $r<R$ such that

$$
\begin{aligned}
& \frac{\left(b^{+}\right)^{*}}{r^{\lambda}}-\frac{\left(h^{-}\right)_{*}}{R^{\lambda}} \leq R, \\
& \frac{\left(h^{+}\right)_{*}}{R^{\lambda}}-\frac{\left(b^{-}\right)^{*}}{r^{\lambda}}+\gamma_{*} \geq r .
\end{aligned}
$$

If we fix $r=r_{0}$ and $R=\frac{\left(b^{+}\right)^{*}}{r_{0}^{\lambda}}$, then $R \geq 1>\frac{1}{2} \geq r$ and (2.22), (2.23) ensure that (2.24) and (2.25) hold.

Remark 2.2 It is easy to check that if $I_{-}^{b}=\emptyset$, then (2.23) reduces to

$$
\frac{(h)_{*}}{\left[(b)^{*}\right]^{\lambda}} r_{0}^{\lambda^{2}}+\gamma_{*} \geq r_{0}
$$

As in the proof of [1, Theorem 4], we may take $r_{0}=\left[\frac{h_{*}}{b^{* \lambda}} \lambda^{2}\right]^{\frac{1}{1-\lambda^{2}}}$, then $(2.26)$ can be reduced by the condition (2.22). 
Remark 2.3 As an application of Theorem 2.1, let us consider the problem

$$
\begin{aligned}
& u^{\prime \prime}(t)+\frac{1}{16} u(t)=\frac{b(t)}{u^{\lambda}(t)}+c(t), \quad t \in(0,1), \\
& u(0)=u(T), \quad u^{\prime}(0)=u^{\prime}(T),
\end{aligned}
$$

where

$$
c(t) \equiv 1, \quad b(t)=t-\frac{1}{10}, \quad t \in[0,1] .
$$

By [28, Lemma 2.3], the Green function of the linear problem

$$
\begin{aligned}
& u^{\prime \prime}(t)+\frac{1}{16} u(t)=0, \quad t \in(0,1), \\
& u(0)=u(T), \quad u^{\prime}(0)=u^{\prime}(T),
\end{aligned}
$$

can be explicitly given by

$$
G(t, s)=\frac{2\left(\sin \frac{t}{4}-\sin \frac{t-1}{4}\right)\left(\sin \frac{s}{4}-\sin \frac{s-1}{4}\right)}{\sin \frac{1}{4}\left(1-\cos \frac{1}{4}\right)}+\frac{4}{\sin \frac{1}{4}} \begin{cases}\sin \frac{t-1}{4} \sin \frac{s}{4}, & 0 \leq s \leq t \leq 1, \\ \sin \frac{s-1}{4} \sin \frac{t}{4}, & 0 \leq t \leq s \leq 1 .\end{cases}
$$

Equation (2.29) yields

$$
\gamma(t)=\int_{0}^{1} G(t, s) c(s) d s=\frac{16\left(\sin \frac{t}{4}-\sin \frac{t-1}{4}\right)}{\sin \frac{1}{4}}+\frac{16}{\sin \frac{1}{4}}\left(\sin \frac{t-1}{4}+\sin \frac{1}{4}-\sin \frac{t}{4}\right)=16
$$

and

$$
\begin{aligned}
\beta^{-}(t)= & \int_{0}^{1} G(t, s) b^{-}(s) d s \\
< & \int_{0}^{\frac{1}{10}} G(t, s) d s \\
= & \frac{8\left(\sin \frac{t}{4}-\sin \frac{t-1}{4}\right)\left(1-\cos \frac{1}{4}+\cos \frac{9}{40}-\cos \frac{1}{40}\right)}{\left(1-\cos \frac{1}{4}\right) \sin \frac{1}{4}} \\
& +\frac{16\left(\sin \frac{t-1}{4}-\cos \frac{9}{40} \sin \frac{t}{4}+\sin \frac{1}{4}\right)}{\sin \frac{1}{4}}
\end{aligned}
$$

and consequently

$$
\gamma_{*}=16, \quad\left(\beta^{-}\right)^{*} \doteq 1.99729 .
$$

Since $1.99729 \leq 8^{1+\lambda}$ for all $\lambda \in(0, \infty)$, Theorem 2.1 guarantees that the set (2.27), (2.28) has at least one positive 1-periodic solution. 


\section{Author's contributions}

YM completed the study, carried out the results of this article and drafted the manuscript, and checked the proofs and verified the calculation. The author read and approved the final manuscript.

\section{Acknowledgements}

This work was supported by the NSFC (No. 11361054).

Received: 25 June 2014 Accepted: 23 July 2014 Published online: 25 September 2014

\section{References}

1. Lazer, AC, Solimini, S: On periodic solutions of nonlinear differential equations with singularities. Proc. Am. Math. Soc. 99, 109-114 (1987)

2. Gordon, WB: Conservative dynamical systems involving strong forces. Trans. Am. Math. Soc. 204, 113-135 (1975)

3. Gordon, WB: A minimizing property of Keplerian orbits. Am. J. Math. 99, 961-971 (1977)

4. Bonheure, D, Fabry, C, Smets, D: Periodic solutions of forced isochronous oscillators at resonance. Discrete Contin. Dyn. Syst. 8(4), 907-930 (2002)

5. Fonda, A: Periodic solutions of scalar second order differential equations with a singularity. Mém. Cl. Sci., Acad. R. Belg. 8, 68-98 (1993)

6. Fonda, A, Manásevich, R, Zanolin, F: Subharmonics solutions for some second order differential equations with singularities. SIAM J. Math. Anal. 24, 1294-1311 (1993)

7. Habets, P, Sanchez, L: Periodic solutions of some Liénard equations with singularities. Proc. Am. Math. Soc. 109, 1135-1144 (1990)

8. Jiang, D, Chu, J, Zhang, M: Multiplicity of positive periodic solutions to superlinear repulsive singular equations. J. Differ. Equ. 211(2), 282-302 (2005)

9. del Pino, M, Manásevich, R: Infinitely many $T$-periodic solutions for a problem arising in nonlinear elasticity. J. Differ. Equ. 103, 260-277 (1993)

10. del Pino, M, Manásevich, R, Montero, A: T-Periodic solutions for some second order differential equations with singularities. Proc. R. Soc. Edinb., Sect. A 120(3-4), 231-243 (1992)

11. del Pino, M, Manasevich, R, Murua, A: On the number of $2 \pi$-periodic solutions for $u^{\prime \prime}+g(u)=s(1+h(t))$ using the Poincaré-Birkhoff theorem. J. Differ. Equ. 95, 240-258 (1992)

12. Rachunková, I, Stanek, S, Tvrdý, M: Singularities and Laplacians in boundary value problems for nonlinear ordinary differential equations. In: Handbook of Differential Equations: Ordinary Differential Equations, vol. 3. Elsevier, Amsterdam (2006)

13. Torres, PJ: Bounded solutions in singular equations of repulsive type. Nonlinear Anal. 32, 117-125 (1998)

14. Torres, PJ, Zhang, M: Twist periodic solutions of repulsive singular equations. Nonlinear Anal. 56, $591-599$ (2004)

15. Zhang, M: A relationship between the periodic and the Dirichlet BVPs of singular differential equations. Proc. R. Soc. Edinb., Sect. A 128, 1099-1114 (1998)

16. Rachunková, I, Tvrdý, M, Vrkoč, I: Existence of nonnegative and nonpositive solutions for second order periodic boundary value problems. J. Differ. Equ. 176, 445-469 (2001)

17. Torres, PJ: Existence of one-signed periodic solutions of some second order differential equations via a Krasnoselskii fixed point theorem. J. Differ. Equ. 190, 643-662 (2003)

18. Torres, PJ: Weak singularities may help periodic solutions to exist. J. Differ. Equ. 232, 277-284 (2007)

19. Franco, D, Webb, JKL: Collisionless orbits of singular and nonsingular dynamical systems. Discrete Contin. Dyn. Syst. $15,747-757$ (2006)

20. Chu, J, Li, M: Positive periodic solutions of Hill's equations with singular nonlinear perturbations. Nonlinear Anal. 69 , 276-286 (2008)

21. Li, X, Zhang, Z: Periodic solutions for damped differential equations with a weak repulsive singularity. Nonlinear Anal. 70(6), 2395-2399 (2009)

22. Ma, R, Chen, R, He, Z: Positive periodic solutions of second-order differential equations with weak singularities. Appl. Math. Comput. 232, 97-103 (2014)

23. Bravo, JL, Torres, PJ: Periodic solutions of a singular equation with indefinite weight. Adv. Nonlinear Stud. 10(4), 927-938 (2010)

24. Hakl, R, Torres, PJ: On periodic solutions of second-order differential equations with attractive-repulsive singularities. J. Differ. Equ. 248(1), 111-126 (2010)

25. Saito, H, Ueda, M: Dynamically stabilized bright solitons in a two-dimensional Bose-Einstein condensate. Phys. Rev. Lett. 90(4), 040403 (2003)

26. Centurion, M, Porter, MA, Pu, Y, Kevrekidis, PG, Frantzeskakis, DJ, Psaltis, D: Modulational instability in a layered Kerr medium: theory and experiment. Phys. Rev. Lett. 97, 234101 (2006)

27. Hau, LV, Burns, MM, Golovchenko, JA: Bound states of guided matter waves: an atom and a charged wire. Phys. Rev. A 45(9), 6468-6478 (1996)

28. Ma, R, Gao, C, Chen, R: Existence of positive solutions of nonlinear second-order periodic boundary value problems. Bound. Value Probl. 2010, Article ID 626054 (2010)

doi:10.1186/s13661-014-0188-

Cite this article as: Ma: Existence of positive periodic solutions of second-order differential equations with weak singularities. Boundary Value Problems 2014 2014:188. 